\title{
The Design of 2DOF Enhance Coordination Unit of Ammunition
}

\author{
Guobao Ding ${ }^{1, a}$, He Cui ${ }^{2, b}$, Xiuwen $\mathrm{Hu}^{3, \mathrm{c}}$ \\ ${ }^{1,2,3,4}$ Armor Technique Institute of PLA, Changchun ,China \\ a294244476@qq.com, ${ }^{\text {b15304459101@189.cn, }{ }^{c} 253389412 @ q q . c o m}$
}

Keywords: 2DOF; enhance coordination unit of ammunition; design

\begin{abstract}
Modern tanks is the development direction of automation and information technology, in order to achieve this goal, reduce the number of crew tanks, protect the life safety of the soldiers, tanks adopts automatic loading system is inevitable trend of ammunition. Automatic ammunition loading system is generally divided into ammunition storehouse, coordinator, lose play machine three parts. Based on the ammunition coordinator in the automatic loading system and lose spring machine as the research object, the design with two degrees of freedom ammunition improved coordination unit of concrete structure.
\end{abstract}

\section{Introduction}

In the future digital tank weapons platform, automatic ammunition loading system is the core part of the mechanical system and key technology. Automatic ammunition loading system in the future must be the standard configuration of tank weapon system. In automatic ammunition loading system, according to the function different, the whole system can be divided into three main parts, automatic ammunition storehouse, respectively, ammunition improved coordination device and TuiDan device. Realization of automatic ammunition storehouse of the automatic loading, unloading and storage of ammunition and choose to ascend and transfer ammunition to the corresponding ammunition coordination unit function of quality. Ammunition improved coordination unit responsible for receiving from ammunition storehouse ammunition, and to transmit them to cannon tail at the back, with the gun axis alignment, by losing machines will be reliable, consistent ammunition to push into the bore. TuiDan device is the ammunition by cannon tail at the back of the rapid and steady input bore, and ensure good orientation consistency under arbitrary beam Angle. Because of the artillery trunnion rotate itself in order to realize the change of the shoot Angle, and the shape of the ammunition for the general for the cylindrical rigid body with a tapered tip, therefore, to remove ammo from ammunition storehouse or pushed into the gun, for ammunition coordination unit in a certain way can with the axis of the projectile ammunition storehouse, in another Angle with the axis of the bore to TuiDan into the chamber[1][2] .

Based on the function of the ammunition improved coordination device, generally can be divided into two functional parts. Respectively responsible for the lift ammunition supporting device and implementation of ammunition position mobile transmission. Considering the single degree of freedom of ammunition the movement of the lifting device is actually a tiny around artillery trunnion rotation motion, and to the projectile loading into the gun, you must request the axis of the projectile and the gun axis coincidence. As a result, the projectile can be around artillery trunnion rotary motion into tiny along the turret vertical translation and the projectile moves around and play tray and the projectile center of gravity of the rotary motion of two degrees of freedom.

\section{Design Thought of 2DOF Enhance Coordination Unit of Ammunition}

Considering projectiles from cylinder movement to the gun need to complete the process of the three different movement action: drawn from the magazine ammunition, coordinated movement in place, to push the projectile into the gun. In traditional ammunition to promote coordinating device, extract process of ammunition from cylinders are usually performed by automatic cylinder, namely a putter in automatic warehouse the end of the installation will be specified by the projectile 
launched from the magazine to the coordinator of the elastic plate, but due to a convex edge at the end of the projectile, so direct to launch the projectile will cause in the process of TuiDan into the chamber, the projectile hemline card in play tray edge, TuiDan into the chamber. Therefore, the existing automatic ammunition for lost play system generally adopts the design of ammunition separation after the first load the projectile load module form. And this kind of design for the lost in double time, directly affect the shooting speed. And in the process of TuiDan into the chamber, the existing automatic ammunition for lost play system generally USES solid even lose play machine make the action in the barrel of the gun, this design makes the cannon tail size increases, the cannon tail length increase of at least one play long, which affects the size of the whole tank turrets, is not conducive to the survival of tanks on the battlefield.

So, considering the existing automatic for lost play these deficiencies in the system, in this paper, considering the projectile bumps the bottom edge, will automatically play to launch the projectile motion and lose machine play TuiDan chamber into the action together, simplifying structure, optimize the structure of turret, and meet the requirements of the antitank shells ready, improve the efficiency of the shooting. But need only existing automatic warehouse of ammunition storage by the tip of the projectile turret to point to the turret tail ahead.

Thus, in this paper, the entire ammunition ascension movement coordination device is divided into five parts, respectively is: institutions from the action of fetching ammunition in drug storehouse, backward motion of extracting ammunition, enhance coordination in place of ammunition improved coordination.

\section{The Function Module Dividing of 2DOF Enhance Coordination Unit of Ammunition}

The improved coordination motion is divided into five movements, the action of fetching ammunition and release ammunition which use the projectile hemline bump, so you can use the same components to complete the two actions. And extracting the projectile movement with practical action of TuiDan into the chamber is along the forward and backward movement of the elastic plate, which use the same components to complete. At the same time, considering the ammunition during transmission need to hold the projectile, an intermediate device is rebounding, and the projectile process of extracting and pushed into the chamber are along the elastic plate, so will grab (release) components, extraction (push) components are arranged at the play on a board divided into a separate module.

Ammunition promote improved coordination of movement coordination device needs a rotational motion and the motion of a moving vertically compound and into, therefore, the movement action need two modules to complete, that is, a rotating module and an ascending module, which turn the module in ascending module leads to complete the movement of the vertical direction, the play tray in turning module leads to complete the action, so as to elevate the projectile coordinate for the location of the bore axis coincidence.

\section{DOF Enhance Coordination Unit of Ammunition}

Play board module consists of three parts, respectively for ammunition fetching (release) module, ammunition extraction (push) module and rebounding module. Is composed of five movements need to be done:

1) institutions fetching drug storehouse of ammunition

2) extracting ammunition backward movement

3) institutions TuiDan such as chamber to move forward

4) agency release the ammunition

5) institutions TuiDan in place

Ammunition fetching (release) module is responsible for the complete action 1 and 4, ammunition extraction (push) module is responsible for the complete action 2 and 3 . And in order to ensure that a bomb in place, so should design a compression device pressing projectile can be forced into the chamber of the institution, ensure TuiDan in place. Now design is as follows, 
respectively.

Ammunition fetching (release) the design of the module.

This article monolithic projectile ammunition improved coordination system adopted, its weight is $35 \mathrm{~kg}$, considering a projectile bottom edge as shown in Fig. 1 of bump, reference machine three catch chuck design one sleeve claw CAM mechanism to realize sports movement.

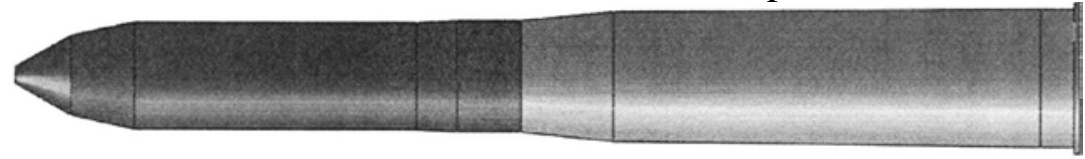

Fig. 1 a grenade

As shown in Fig.2 and Fig.3, jaw with claw hook, installed in the jaw plate with the outer surface of the jaw plate groove, just can get stuck projectile hemline. At the same time, can move along the jaw plate of the radial of jaw, thus completed scraping or release the projectile motion. In the jaw of a raised small tail shaft installed in the jaw plate CAM slot, when the CAM rotation Angle, a groove along the jaw jaw plate movement, so as to realize the ammunition to grab and release.

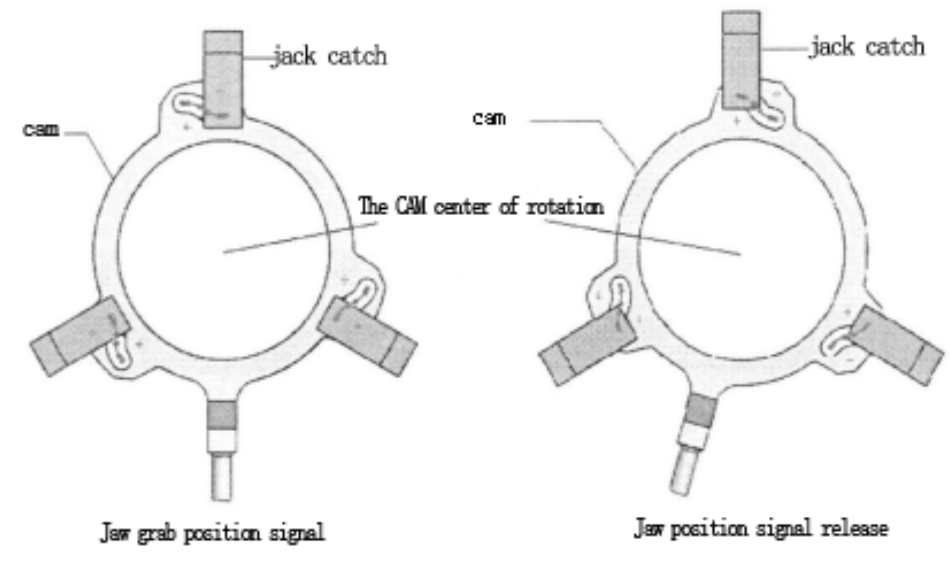

Fig.2 Jaw movement

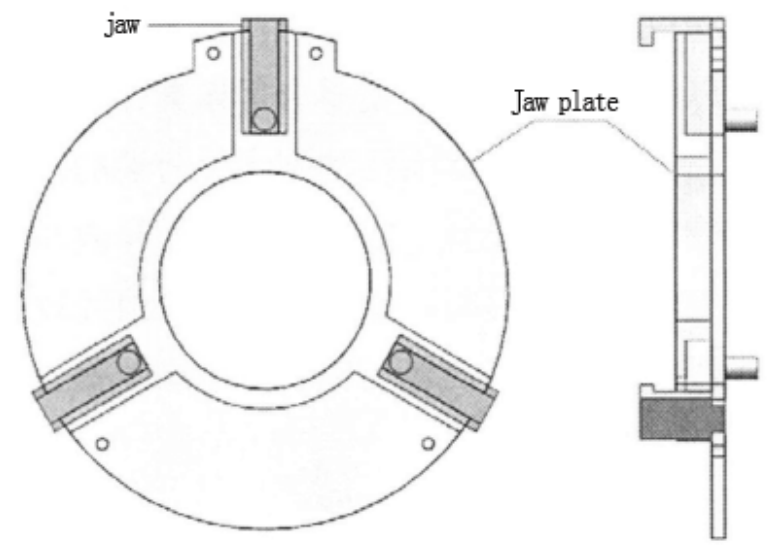

Fig.3 Jaw and jaw plate of schematic diagram

To achieve the rotation of the CAM revolves around the center of rotation, considering projectiles along the disk guide with lateral displacement, and the projectile to grab and release action are completed in the front of the play tray of action. Therefore, on the CAM design a swing arm, on the play tray guideway design a chute to accommodate the swing arm. The front of the guide rail groove for a curved groove, and guide the motion of the swing arm, for the rest of the line, used to lock the CAM, so as to make the jaw grasp the projectile. As shown in figure 4, the swing arm installed inside a guide groove CAM, CAM by the end of a guide rail movement to put in place when the release of the front/grab, the crank CAM due to the shape change of guide grooves around the rotation Angle, so as to release/contraction jaw.

When considering the crank CAM rotation around the center of rotation, in space across the 
curve is relatively complex, high processing cost, so the tilting mechanism instead of guide groove guide CAM rotation. Its structure is shown in Fig.5.

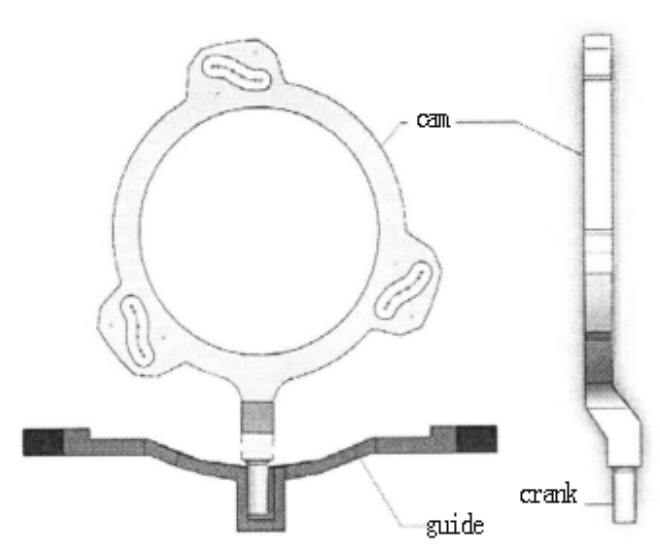

Fig.4 The CAM motion diagram

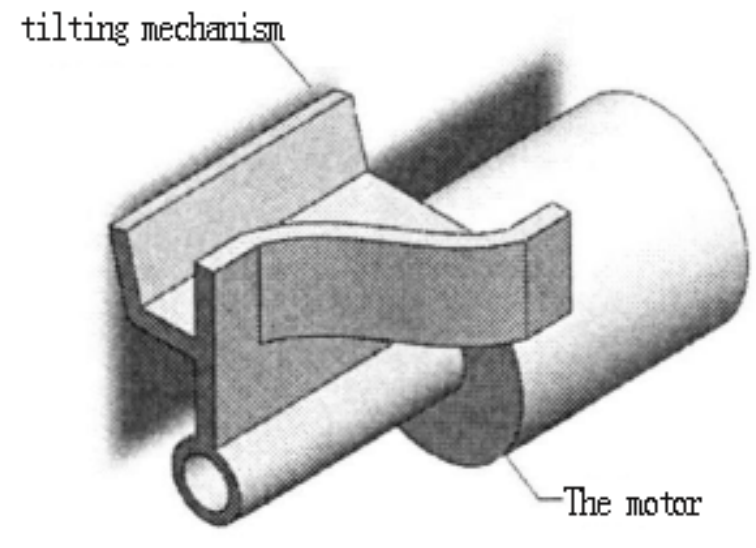

Fig.5 Tilting mechanism

Locking CAM when losing play, motor crank up along one edge of the tilting mechanism surface movement in the process of complete swing motion, when extracting ammunition, motor rotation, compression crank CAM to jaw contraction position, grasping the ammunition.

Ammunition extraction (push) module design.

In the process of extraction with ammunition and ammunition displacement is greater than a play long, namely S > $1250 \mathrm{~mm}$. Displacement is larger, so the complete movement action chain drive way. As shown in Fig.6.

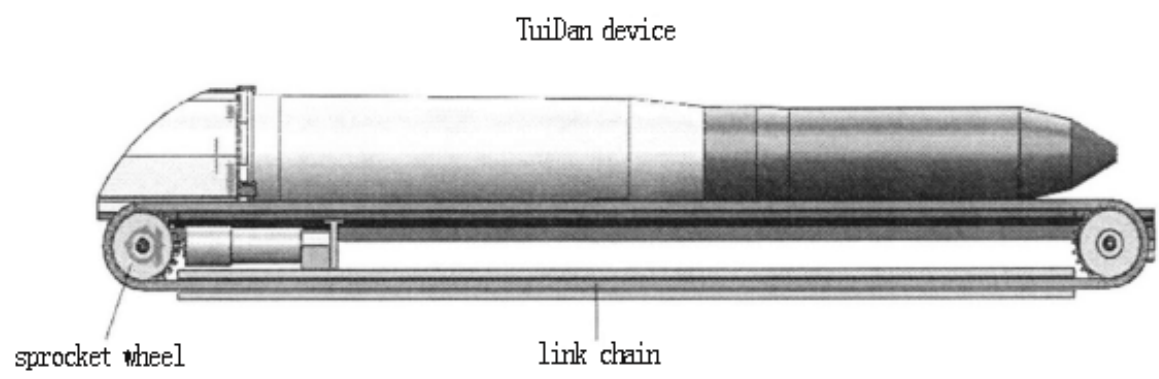

Fig.6 Chain transmission sketch

To save space structure, to avoid movement interference will decorate below the guide rail used chain drive motor. The motor output direction and sprocket wheel rotation direction orthogonal rotary motion, thus orthogonal bevel gear to convert the output direction to the chain wheel rotation direction. As shown in Fig.7.

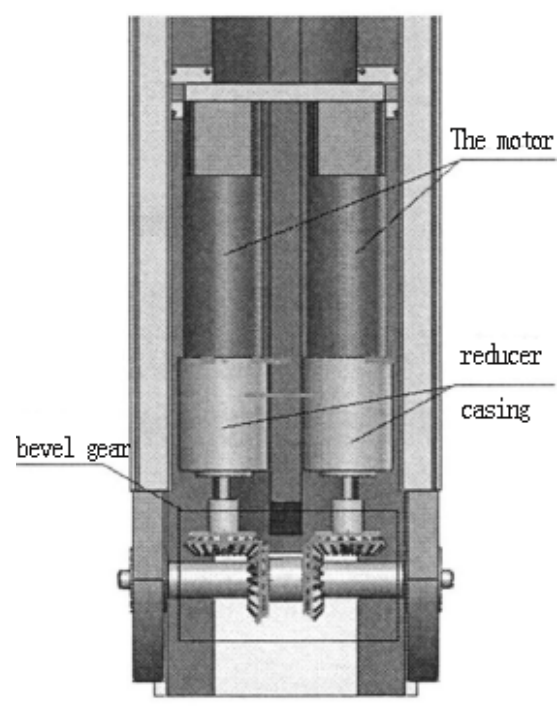

Fig.7 Bevel gear schematic 


\section{Rotating part of the design}

\section{The moment of inertia is calculated.}

Given in loads and moment of inertia of the rotating parts, and the calculation of moment of inertia is very complex, so the agency by $3 \mathrm{~d}$ software modeling, and then simulated. This article USES the solidworks model and measured TuiDan part of the rotational inertia of the axis of rotation.

Step 1: on the modeling components.

Step 2: the material editor.

Moment of inertia of the distribution of the influencing factors of quality and quality, if the first step in the modeling accuracy, it can guarantee the correctness of the volume, in the material editor this step, to edit the is actually a material density p, in solidworks software has a built-in material database, you can find the corresponding brand of domestic materials, in the database, so need to check the manual and other means to calculate density p values, then separate editorial material properties.

Step 3: simulation assembly.

Step 4: defining coordinate system. Device at work based on the actual rotor shaft or required by the theory, to found in the assembly and the matching position, and then to establish a coordinate system, and name, as shown in Fig.8.

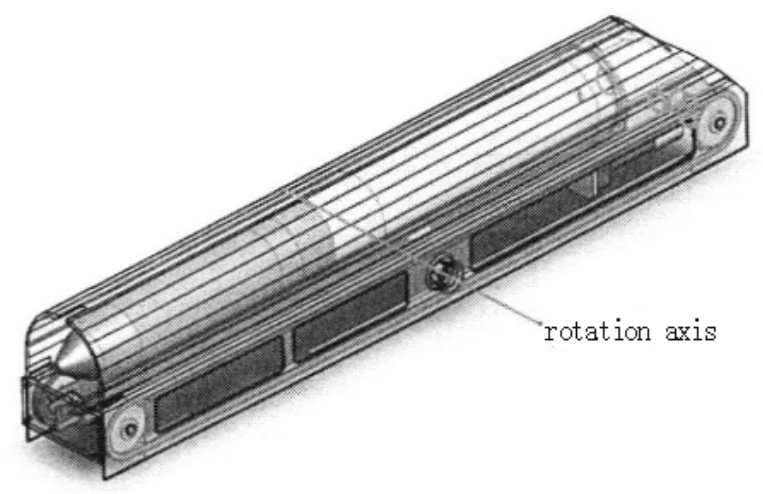

Fig.8 rotating coordinate system schematic diagram

Step 5: simulation calculation and record data. In the dialog box to select the fourth step defined in the coordinate system, click the calculate button can get results. Specific results are as follows:

The quality is $47275.00 \mathrm{~g}$,Volume is $31397907.71 \mathrm{~mm}^{3}$.

Surface area is 4453057.54 .

The center of gravity $(\mathrm{mm}): \mathrm{X}=-0.05, \mathrm{Y}=91.16, \mathrm{Z}=0.51$.

The inertial principal axis and inertia moment: $g *$ was, decided by the center of gravity.

$\mathrm{Ix}=0.00,0.01,1.00$

$\mathrm{Iy}=-0.01,-1.00,0.01$

$\mathrm{Iz}=1.00,-0.01,0.00$

Inertia tensor is determined by the center of gravity, and alignment of the output coordinate system.

Lxx,Lxy,Lxz=14872676141.48,459267.33,-3513392.38

Lyx,Lyy,Lyz=459267.33,14776465918.37,165553526.56

Lzx,Lzv,Lzz=-3513392.38,165553526.56,591969596.14

Inertia tensor is determined by the coordinate system.

$\mathrm{Px}=590036749.39$

$\mathrm{PY}=14778395249.50$

$\mathrm{Pz}=14872679657.10$

Ixx,lxy,Ixz=7680661947.42,401030.295612,1923361.48794

Iyx,Iyy,Iyz=401030.295612,6712781975.15,-2426844633.76

Izx,Izy,Izz=1923361.48794,-2426844633.76,1210403357-27

The Ixx to ask around the center of rotation of the moment of inertia. 


\section{The Design of Ascending Part}

Projectile and the distance from the lowest to the highest position for the $\mathrm{S}=1280.84 \mathrm{~mm}$, add quality attributes of the established $3 \mathrm{~d}$ model, the quality can be measured in the SolidWorks model, total quality be promoted by the measurement of about: $m=52.27 \mathrm{~kg}$. Load should be followed: Fg $=\mathrm{mg}=512.246 \mathrm{~N}$, assigned to the article on both sides of the load should be: $\mathrm{Fa}=0.5 \mathrm{Fg}=$ 256.123 N 256.2 N material. According to design requirements, should be in 1.5 seconds will lose institutions in place, the average speed should be followed: $\mathrm{Ve}=0.853 \mathrm{~m} / \mathrm{s}$. Considering the start-stop movement process, computing speed is: $n=\mathrm{lm} / \mathrm{s}$. By querying the motor manual, select MAXON's EC60 type brushless dc motor, its rated power is $400 \mathrm{w}$.

\section{Conclusion}

For tank guns and ammunition automatic loading system for its overall performance, especially the fire performance, operational capability and battlefield survival ability has a vital role, and ammunition improved coordination unit to a large extent determines the whole automatic loading system, the overall performance of the ammunition. This paper proposes a two degrees of freedom of ammunition improved coordination units, and according to the specific requirements designed with two degrees of freedom ammunition improved coordination unit of the main components. By manual refer to mechanical design and motor selection manual, ammunition, enhance coordination requirement of track, speed and so on, and put forward, including dc motor, gear reducer, chain drive, power transmission system, and according to the established $3 \mathrm{~d}$ model proposed solutions.

In order to further explore two degrees of freedom mechanical and electrical characteristics of ammunition improved coordination device, two degrees of freedom in practical application of ammunition improved coordination device, the establishment of a two degrees of freedom ammunition improved coordination device physical scale model, some experiments. Experiments proved that this design of two degrees of freedom ammunition on the design principle of the feasibility of the improved coordination device. At the same time, through the establishment of dynamic model of scale model, and the joint simulation model, proves the feasibility of PID control method is adopted in this paper.

\section{References}

[1] Baolin Hou,Junmou Qiao,Qiongmin Liu.Artillery automatic loading [M].Beijing: weapon industry press, 2010 .

[2] Lebin Tan,Xiangyan Zhang.Introduction to artillery [M]. Nanjing university of science and technology.

[3] Krauss-Maffei Wegmann GmbH\&CO.KG.Full Spectrum Artillery Indirect Fire Support[M]. Munich:Krauss-Maffei Wegmann GmbH\&CO.KG,2008.

[4] BADDELEY ADAM.Enhanced Range,Reduced Cost[J].Land Warfare.2009(2):20.

[5] Zhong Jin.Tank guns Autoloader [J]. Foreign tanks,2004(3):44-45.

[6] Hongchao Han.South Africa T6 type turret automatic loading system [J]. Journal of artillery launch and control. 2010(2).

[7] TOLBERTMAJOR VINCENT J.NLOS Cannon:meeting the demandsoffuture combat[J]. Field Artillery, 2006(3-4):10-12. 